\title{
Estimativa da produção de sedimentos mediante uso de um modelo hidrossedimentológico acoplado a um SIG
}

\author{
$\overline{\text { Richarde M. da Silva }{ }^{1} \& \text { Celso A. G. Santos }}{ }^{2}$
}

\begin{abstract}
RESU MO
0 Kineros é um modelo hidrossedimentológico usado para se estimar a vazão e a produção de sedimentos em bacias urbanas e rurais. No presente trabalho, o modelo foi aplicado à bacia do rio Pirapama, localizada na zona litorânea do Estado de Pernambuco, cujos resultados foram acoplados a um SIG com o objetivo de identificar, temporal e espacialmente, os principais locais em que o processo de erosão foi mais atuante na bacia do rio Pirapama. 0 model o foi calibrado com dados diários de 1990 a 2001 de dois postos pluviométricos. Através do acoplamento dos resultados da modelagem no SIG, foi possível a identificação das principais áreas susceptíveis ao processo erosivo. 0 s resultados da produção de sedimentos na bacia mostraram que os planos com maior produção produziram mais de $200 \mathrm{tha}^{-1} \mathrm{ano}^{-1}$, perfazendo 0 total de $653.079 \mathrm{t}$, correspondentes a uma área de $67,87 \mathrm{~km}^{2}$ (11,3\% da área total da bacia) revelando, assim, que grande parte da bacia é realmente susceptivel ao processo de erosão. Este trabalho mostra que o modelo Kineros é viável para bacias maiores que $100 \mathrm{~km}^{2}$ e 0 seu acoplamento ao SIG é de grande valia para a identificação e análise das principais áreas de produção de sedimentos na bacia deste rio podendo ser, assim, considerado ferramenta promissora para simulação da produção de sedimentos em bacias hidrográficas do nordeste brasileiro.
\end{abstract}

Palavraschave: modelagem, Kineros, modelo físico, Bacia Pirapama

\section{Sediment yield prediction through the use of a runoff-erosion model coupled to a GIS}

\begin{abstract}
Kineros is a runoff-erosion model used to compute runoff and sediment yield in urban and rural basins. In this paper, the model was applied to the Pirapama river basin, located in the coastal zone of the State of Pernambuco. The obtained results were linked to a GIS in order to temporally and spatially identify the areas susceptible to the erosion process within the Pirapama river basin. The model was calibrated with daily rainfall data from two raingauges for the period from 1990 to 2001. From the coupling of the modeling results into a GIS, it was possible to identify the main areas susceptible to the erosion process. The sediment yield results showed that the planes with the largest erosion yield produced more than $200 \mathrm{t}$ ha $^{-1}$ year ${ }^{1}\left(653,079\right.$ tin an area of $67.87 \mathrm{~km}^{2}, 11.3 \%$ of the total area). These results also showed that a large part of the basin is indeed susceptible to the erosion process. This study shows that the Kineros model is applicable to basins larger than 100 $\mathrm{km}^{2}$ and that its coupling to a GIS proved useful to identify and analyze the main areas with sediment yield within the basin. Thus, the model can be considered as a promising tool for simulation of the sediment yield in basins in northeastern Brazil.
\end{abstract}

Key words: modeling, Kineros, physically-based model, Pirapama Basin

\footnotetext{
1 Departamento de Geociências/U FPB. CEP 58051-900, João Pessoa, PB. Fone (83) 3216-7432. E-mail: richardemarques@yahoo.com.br 2 Departamento de Engenharia Civil e Ambiental/U FPB, CEP 58051-900, João Pessoa, PB. Fone/Fax (83) 3216-7684. E-mail: celso@ct.ufpb.br
} 


\section{INTRODUÇÃO}

A erosão é uma importante questão ambiental que afeta diretamente a sociedade, reduzindo a capacidade de reservatórios, intensificando o transporte de poluentes agregados às partículas de sedimentos (Araújo \& Knight, 2005) e reduzindo o potencial da produção agrícola em áreas agricultáveis, devido aos processos de desprendimento e arraste de partículas (Firouzabadi \& Davoodi, 2004).

Nas últimas décadas, modelos distribuídos de base física têm sido intensamente utilizados em estudos de avaliação do comportamento e impactos das mudanças do uso do solo e suas influências nos processos de escoamentos superficial e subterrâneo, desprendimento, transporte e deposição de sedimentos em bacias hidrográficas. Recentemente, modelos hidrossedimentológicos de base física vêm sendo cada vez mais aplicados na medição e na estimativa da produção de sedimentos (Figueiredo \& Bathurst, 2002) em escalas cada vez maiores, trazendo significativas contribuições para a gestão dos recursos hídricos.

Nos últimos anos, o número de estudos hidrossedimentológicos desenvolvidos no Brasil aumentou consideravelmente devido ao fato da relevância dada a esse tema, por diversos pesquisadores, no País e no exterior. Santos et al. (2000) desenvolveram uma equação empírica de perda de solo para a região semi-árida do nordeste brasileiro baseada nos resultados de um modelo hidrossedimentológico de base física, todavia, ainda são raros os estudos aplicados à quantificação da produção de sedimentos em bacias hidrográficas com mais de $100 \mathrm{~km}^{2}$, principalmente em bacias localizadas no litoral nordestino, como é o caso da bacia do rio Pirapama.

Desde 2001, a bacia Pirapama é uma das principais fontes de abastecimento de água da Região Metropolitana do Recife mas enfrenta, hoje, sério problema com a degradação ambiental generalizada, acompanhada de contínuos desmatamentos de áreas florestais, associados aos processos de ocupação do território para habitação e para o desenvolvimento de atividades agroindustriais, como uma vasta extensão de sua área destinada ao plantio de cana-de-açúcar, portanto, devido a tais fatores, estudos de estimativa da produção de sedimentos são urgentes para a identificação de áreas mais susceptíveis à produção de sedimentos, principalmente nas bacias do litoral nordestino com mais de $100 \mathrm{~km}^{2}$.

Nos últimos anos, as aplicações de modelos hidrossedimentológicos vêm sendo incorporadas ao Sistema de Informações Geográficas (SIG), para representar espacialmente os resultados obtidos da modelagem, sobretudo na produção de mapas temáticos e para auxiliar as análises espaciais, através do cruzamento de dados tabulares e de informações geográficas ajudando, assim, o processo de análise geoespacial.

Lima et al. (1992) verificaram, em área teste da Paraíba, a susceptibilidade natural e o risco de erosão por intermédio do uso de um software SIG, desenvolvido especificamente para Sensoriamento Remoto, e da Equação Universal da Perda de Solo, e mencionaram que a principal característica do estudo foi a possibilidade de se estimar, a partir de imagens de satélite, o risco de erosão em grandes áreas.
Kalin et al. (2003) estimaram a vazão e a produção de sedimentos através do acoplamento entre o modelo Kineros e um SIG, com a sobreposição de mapas físicos da bacia para a obtenção da rede de fluxo, do delineamento da área de drenagem e da declividade média para a parametrização automatizada de parâmetros físicos de duas microbacias experimentais mas não demonstraram os resultados obtidos em mapas temáticos.

Desta forma, o presente trabalho tem como objetivo quantificar, temporal e espacialmente, a produção de sedimentos na bacia do rio Pirapama, através do acoplamento entre o modelo hidrossedimentológico de base física Kineros e um SIG para ajudar na identificação e representação espacial das áreas da bacia mais susceptíveis à erosão; para tanto, o modelo foi descrito, o procedimento de sua calibração foi discutido, o índice de erosividade das chuvas para o período estudado, foi analisado e, finalmente, os resultados para as simulações de 1990 a 2001 foram apresentados.

\section{MATERIAL E MÉTODOS}

\section{O modelo hidrossedimentológico}

O modelo escolhido para ser aplicado neste estudo da modelagem da produção de sedimentos da bacia Pirapama, foi o modelo Kineros - Kinematic Runoff and Erosion Model, versão 2 (Woolhiser et al., 1990), uma vez que foi desenvolvido para simular a resposta hidrossedimentológica de bacias urbanas e rurais e já foi calibrado com sucesso para uma região do litoral nordestino (Santos et al., 2004), além de ter sido testado com sucesso por Santos et al. (2001), para áreas bem maiores que as microbacias reportadas por Kalin et al. (2003).

O Kineros é um modelo hidrossedimentológico cinemático, distribuído e de base física, que usa uma rede de planos e canais que representam os cursos d'água na bacia; sendo assim, a bacia é representada por uma cascata de planos e canais. As equações diferenciais que descrevem o escoamento superficial, o transporte de sedimentos e a deposição de sedimentos nesses planos e canais, são resolvidas pela técnica de diferenças finitas.

\section{Infiltração do solo}

O modelo possui um algoritmo de infiltração que permite uma aproximação física para a redistribuição da água no solo, incluindo a capacidade de recuperação de infiltração durante períodos de estiagens e determinando o percentual de infiltração depois deste período sem chuva. O modelo de infiltração descreve a capacidade de infiltração $f_{c}$ como uma função da profundidade infiltrada I, e necessita de quatro parâmetros básicos para descrever as propriedades de infiltração, que são (a) condutividade hidráulica efetiva saturada do solo $\mathrm{K}_{\mathrm{s}}\left(\mathrm{m} \mathrm{s}^{-1}\right)$; (b) capilaridade integral $\mathrm{G}(\mathrm{m})$; (c) porosidade $\phi$; e (d) índice de distribuição dos tamanhos dos poros $\lambda$; há, também, um parâmetro opcional $\mathrm{C}_{\mathrm{v}}$, o qual descreve a variação aleatória no espaço das propriedades hidráulicas do solo, e uma variável relacionada ao evento, 
denominada saturação relativa inicial da camada superior do solo $S_{\mathrm{i}}$, definida como $\theta_{\mathrm{i}} / \phi$, na qual $\theta_{\mathrm{i}}$ é a umidade inicial do solo. $O$ modelo geral para a infiltrabilidade $\mathrm{f}_{\mathrm{c}}\left(\mathrm{m} \mathrm{s}^{-1}\right)$ é dado como função da profundidade infiltrada $\mathrm{I}(\mathrm{m})$, por:

$$
\mathrm{f}_{\mathrm{c}}=\mathrm{K}_{\mathrm{s}}\left[1+\frac{\alpha}{\mathrm{e}^{\alpha / / \mathrm{B}}-1}\right]
$$

donde B é $(\mathrm{G}+\mathrm{h})\left(\theta_{\mathrm{s}}-\theta_{\mathrm{i}}\right)$, combinando os efeitos da franja capilar $\mathrm{G}(\mathrm{m})$, altura do escoamento $\mathrm{h}(\mathrm{m})$, e a capacidade de armazenamento unitária $\Delta \theta=\left(\theta_{\mathrm{s}}-\theta_{\mathrm{i}}\right)$, na qual $\theta_{\mathrm{s}}$ é a umidade de saturação do solo. $O$ parâmetro a representa o tipo de solo, i.e, próximo de 0 para areia, neste caso, a Eq. 1 se aproxima da equação de Green-Ampt, e $\alpha$ é próximo de 1 para um solo franco misto; em tal caso, a Eq. 1 representa a equação de infiltração de Smith-Parlange. Como já mencionado, neste modelo existe uma redistribuição de água no solo pois se considera que, havendo uma estiagem prolongada durante o evento de chuva, o solo deve secar. O método de redistribuição/reinfiltração usado no modelo é descrito em Smith et al. (1993), como também em Corradini et al. (1994).

\section{Erosão nos planos e canais}

A equação geral usada para descrever a dinâmica dos sedimentos em qualquer ponto é dada pelo balanço de massa similar ao escoamento cinemático da água nos planos:

$$
\frac{\partial\left(\mathrm{AC}_{\mathrm{s}}\right)}{\partial \mathrm{t}}+\frac{\partial\left(\mathrm{QC}_{\mathrm{s}}\right)}{\partial \mathrm{x}}-\mathrm{e}(\mathrm{x}, \mathrm{t})=\mathrm{q}_{\mathrm{s}}(\mathrm{x}, \mathrm{t})
$$

donde $\mathrm{C}_{\mathrm{s}}$ é a concentração de sedimentos $\left(\mathrm{m}^{3} \mathrm{~m}^{-3}\right)$, Q é a taxa vazão $\left(\mathrm{m}^{3} \mathrm{~s}^{-1}\right)$, A é a área da seção transversal do escoamento $\left(\mathrm{m}^{2}\right)$, e é a taxa de erosão do solo do leito $\left(\mathrm{m}^{2} \mathrm{~s}^{-1}\right)$, e $\mathrm{q}_{\mathrm{s}}$ é a taxa de entrada lateral de sedimentos nos canais $\left(\mathrm{m}^{2} \mathrm{~s}^{-1}\right)$. Para os planos, e é assumida como sendo composta de dois principais componentes, isto é, pela produção de solo erodido pelos impactos das gotas de chuva sobre o solo descoberto e pela erosão hidráulica (ou deposição) devido à interação entre a força de cisalhamento da água no solo solto do leito e a tendência das partículas do solo se sedimentarem sob a força da gravidade. A taxa total de erosão é um somatório da taxa de erosão pelo impacto das gotas de chuva $\mathrm{e}_{\mathrm{s}} \mathrm{e}$ a taxa de erosão hidráulica $\mathrm{e}_{\mathrm{h}}$ :

$$
\mathrm{e}=\mathrm{e}_{\mathrm{s}}+\mathrm{e}_{\mathrm{h}}
$$

A taxa de erosão pelo impacto da chuva é estimada como:

$$
\mathrm{e}_{\mathrm{s}}=\mathrm{c}_{\mathrm{f}} \mathrm{e}^{-\mathrm{c}_{\mathrm{h}} \mathrm{h}} \mathrm{r}^{2}
$$

na qual $\mathrm{r}$ é a chuva efetiva $\left(\mathrm{m} \mathrm{s}^{-1}\right), \mathrm{c}_{\mathrm{f}}$ é uma constante relacionada ao solo e às propriedades da superfície, $\mathrm{h}$ é a altura do escoamento (m), e $\mathrm{e}^{-c_{\mathrm{h}} \mathrm{h}}$ é um fator de redução, representando a redução na erosão causada pelo aumento da altura da água. $\mathrm{O}$ parâmetro $\mathrm{c}_{\mathrm{h}}$ representa a efetividade do molhamento da água superficial, assumido como sendo igual a 364,0. A taxa de erosão hidráulica $\left(\mathrm{e}_{\mathrm{h}}\right)$ é estimada como sendo linearmente dependente da diferen- ça entre a concentração de equilíbrio e a concentração corrente de sedimento e é dada por:

$$
\mathrm{e}_{\mathrm{h}}=\mathrm{c}_{\mathrm{g}}\left(\mathrm{C}_{\mathrm{m}}-\mathrm{C}_{\mathrm{s}}\right) \mathrm{A}
$$

em que $\mathrm{C}_{\mathrm{m}}$ é a concentração na capacidade de transporte em equilíbrio, $\mathrm{C}_{\mathrm{s}}=\mathrm{C}_{\mathrm{s}}(\mathrm{x}, \mathrm{t})$ é a concentração de sedimento local corrente, e $\mathrm{c}_{\mathrm{g}}$ é um coeficiente de taxa de transferência $\left(\mathrm{s}^{-1}\right)$, que é computado por:

$$
\begin{aligned}
& \mathrm{c}_{\mathrm{g}}=\mathrm{C}_{\mathrm{o}} \frac{\mathrm{v}_{\mathrm{S}}}{\mathrm{h}} \text { se } \mathrm{C}_{\mathrm{s}} \leq \mathrm{C}_{\mathrm{m}} \text { (erosão) } \\
& \text { ou } \mathrm{c}_{\mathrm{g}}=\frac{\mathrm{v}_{\mathrm{S}}}{\mathrm{h}} \text { se } \mathrm{C}_{\mathrm{s}}>\mathrm{C}_{\mathrm{m}} \text { (deposição) }
\end{aligned}
$$

sendo $\mathrm{C}_{\mathrm{o}} \mathrm{o}$ coeficiente de coesão do solo e $\mathrm{v}_{\mathrm{s}}$ é a velocidade de queda da partícula $\left(\mathrm{m} \mathrm{s}^{-1}\right)$.

O modelo usa a relação da capacidade de transporte de Engelund \& Hansen (1967), e a velocidade de queda da partícula é calculada pela seguinte equação:

$$
\mathrm{v}_{\mathrm{s}}^{2}=\frac{4}{3} \frac{\mathrm{g}\left(\rho_{\mathrm{s}}-1\right) \mathrm{d}}{\mathrm{C}_{\mathrm{D}}}
$$

na qual g é a aceleração gravitacional $\left(\mathrm{m} \mathrm{s}^{-2}\right), \rho_{\mathrm{s}}$ é a densidade relativa do sedimento, igual a 2,65 , d é o diâmetro do sedimento $(\mathrm{m})$, e $\mathrm{C}_{\mathrm{D}}$ é coeficiente de resistência da partícula. O coeficiente de resistência da partícula é uma função do número de Reynolds:

$$
\mathrm{C}_{\mathrm{D}}=\frac{24}{\mathrm{R}_{\mathrm{n}}}+\frac{3}{\sqrt{\mathrm{R}_{\mathrm{n}}}}+0,34
$$

sendo $R_{n}$ o número de Reynolds, dado por $R_{n}=v_{s} d / v$, donde $v$ é a viscosidade cinemática da água $\left(\mathrm{m}^{2} \mathrm{~s}^{-1}\right)$. As equações de erosão citadas são aplicadas para cada um dos cinco tamanhos de classe das partículas, que são usadas para descrever o solo quando existir uma variação do tamanho das mesmas. As Eq. 2-8 são resolvidas numericamente para cada espaço de tempo usado nas equações de escoamento e para cada classe de tamanho de partícula.

\section{Determinação da erosividade da chuva}

A erosividade da chuva foi obtida pela Eq. 9 de Lombardi Neto \& Moldenhauer (1992), através de dados da precipitação média mensal calculada pelo Método de Thiessen.

$$
\mathrm{EI}_{\text {mensal }}=89,823\left(\mathrm{P}_{\mathrm{m}}^{2} / \mathrm{P}_{\mathrm{a}}\right)^{0,759}
$$

no qual $\mathrm{EI}_{\text {mensal }}$ é a média mensal do índice de erosão (MJ $\mathrm{mm} \mathrm{ha} \mathrm{h}^{-1} \mathrm{~h}^{-1}$ ) para o mês considerado, $\mathrm{P}_{\mathrm{m}}$ é a precipitação mensal (mm) também do mês considerado, e $\mathrm{P}_{\mathrm{a}}$ é a precipitação média anual (mm).

\section{Calibração do modelo}

A bacia do rio Pirapama foi discretizada em 267 elementos, dos quais 174 são planos e 93, canais. Cada elemento plano foi definido levando-se em consideração a homogeneidade das características dos solos e da declividade média, e as linhas de fluxo. 
Foram utilizados dados diários observados de precipitação dos postos Pirapama e Engenho Cachoeirinha, localizados na bacia, sob responsabilidade do Laboratório de Meteorologia do Estado de Pernambuco, e da Agência Nacional de Águas. O modelo foi calibrado para que os resultados de vazão obtidos se aproximassem das vazões observadas de quatro postos fluviométricos (Ponte PE-35, Destilaria Inexport, Pirapama e Destilaria Bom Jesus).

Como se trata de um modelo de base física, os parâmetros de: (a) porosidade, (b) condutividade hidráulica satura$\mathrm{da}$, e (c) potencial de capilaridade, foram determinados tomando-se como base as características físicas dos solos dos horizontes A e B, para cada plano discretizado da bacia, e baseados nos valores fornecidos por Rawls et al. (1982) para cada tipo de textura de solo. Outros parâmetros, como do percentual de cobertura vegetal, declividade média dos planos e comprimento dos canais, foram obtidos através de mapas digitais da bacia, utilizando-se técnicas da Cartografia Digital.

A Tabela 1 apresenta o intervalo dos valores dos parâmetros ajustados apenas para as duas camadas de solo usadas para a calibração dos parâmetros do modelo. Devido ao fato da bacia possuir camadas de solo muito profundos, constituída de solos do tipo Latossolo Vermelho-Amarelo e Podzólico Vermelho-Amarelo (Silva, 2005) e também por se tratar de uma aplicação com um modelo de base física, optou-se

Tabela 1. Variação dos valores dos parâmetros usados para as camadas superior e inferior de solo

\begin{tabular}{lccc}
\hline Parâmetros & Símbolo & Camada superior & Camada inferior \\
\hline $\begin{array}{l}\text { Capilaridade média do solo } \\
\text { Condutividade hidráulica }\end{array}$ & $\mathrm{G}$ & $20-46 \mathrm{~mm}$ & $12-23 \mathrm{~mm}$ \\
saturada do solo & $\mathrm{K}_{\mathrm{s}}$ & $3-3,6 \mathrm{~mm} \mathrm{~h}^{-1}$ & $0,5-0,8 \mathrm{~mm} \mathrm{~h}^{-1}$ \\
$\begin{array}{l}\text { Fração volumétrica de } \\
\text { rocha }\end{array}$ & $\mathrm{R}_{0}$ & $0,1-0,2$ & $0,1-0,2$ \\
$\begin{array}{l}\text { Porosidade } \\
\text { Espessura da camada }\end{array}$ & $\phi$ & $0,15-0,45$ & $0,13-0,25$ \\
$\begin{array}{l}\text { superior de solo } \\
\text { Espaçamento médio da }\end{array}$ & $\mathrm{H}$ & $300-550 \mathrm{~mm}$ & - \\
$\begin{array}{l}\text { microtopografia } \\
\text { Altura da interceptação }\end{array}$ & $\mathrm{S}_{\mathrm{p}}$ & $0,1-0,3 \mathrm{~m}$ & - \\
$\begin{array}{l}\text { Saturação inicial do solo } \\
\text { Fração da cobertura vegetal }\end{array}$ & $\mathrm{I}_{\mathrm{n}}$ & $0,76-0,90 \mathrm{~mm}$ & - \\
$\begin{array}{l}\text { Coeficiente de rugosidade } \\
\text { de Manning }\end{array}$ & $\mathrm{C}$ & $0,4-0,9$ & - \\
\hline
\end{tabular}

Tabela 2. Produção total de sedimentos, calculada no exutório da bacia Pirapama

\begin{tabular}{|c|c|c|c|c|c|}
\hline Anos & $\begin{array}{l}\text { Produção } \\
\left.\text { (t ha }{ }^{-1} \text { ano-1) }^{-1}\right)\end{array}$ & $\underset{(\mathrm{mm})}{\mathrm{Pm}}$ & Anos & $\begin{array}{l}\text { Produção } \\
\left(\text { t ha }^{-1} \text { ano }^{-1}\right)\end{array}$ & $\underset{(\mathrm{mm})}{\mathrm{Pm}}$ \\
\hline 1990 & 70,03 & 1.910 & 1996 & 80,75 & 2.047 \\
\hline 1991 & 41,77 & 1.194 & 1997 & 87,36 & 1.892 \\
\hline 1992 & 97,20 & 1.975 & 1998 & 36,71 & 1.224 \\
\hline 1993 & 27,34 & 727 & 1999 & 49,37 & 1.375 \\
\hline 1994 & 88,17 & 1.937 & 2000 & 276,44 & 3.401 \\
\hline 1995 & 96,53 & 2.027 & 2001 & 43,39 & 1.463 \\
\hline
\end{tabular}

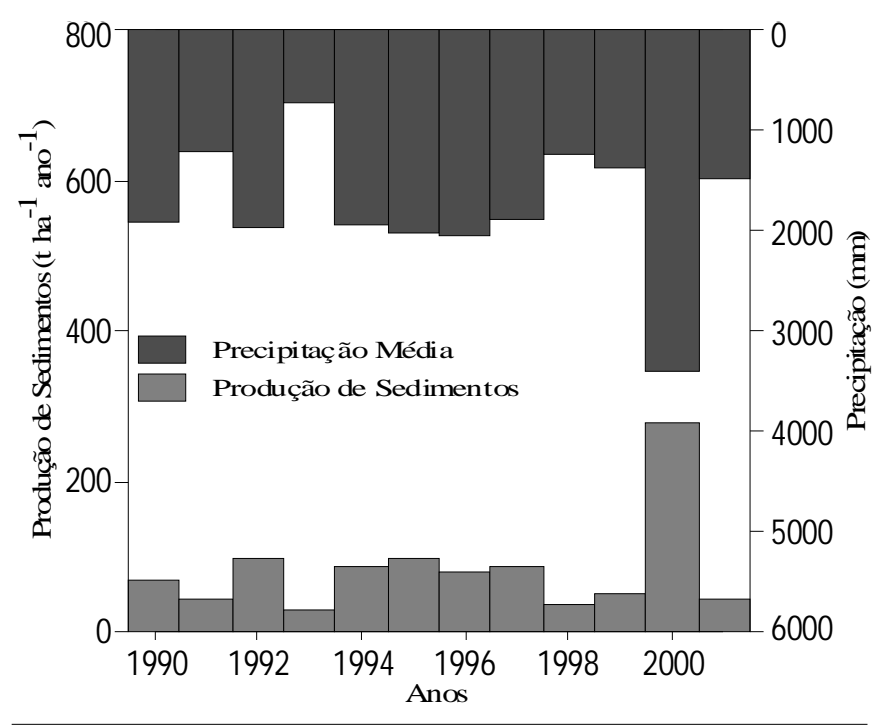

Figura 1. Histograma anual observado e produção total anual calculada de sedimentos

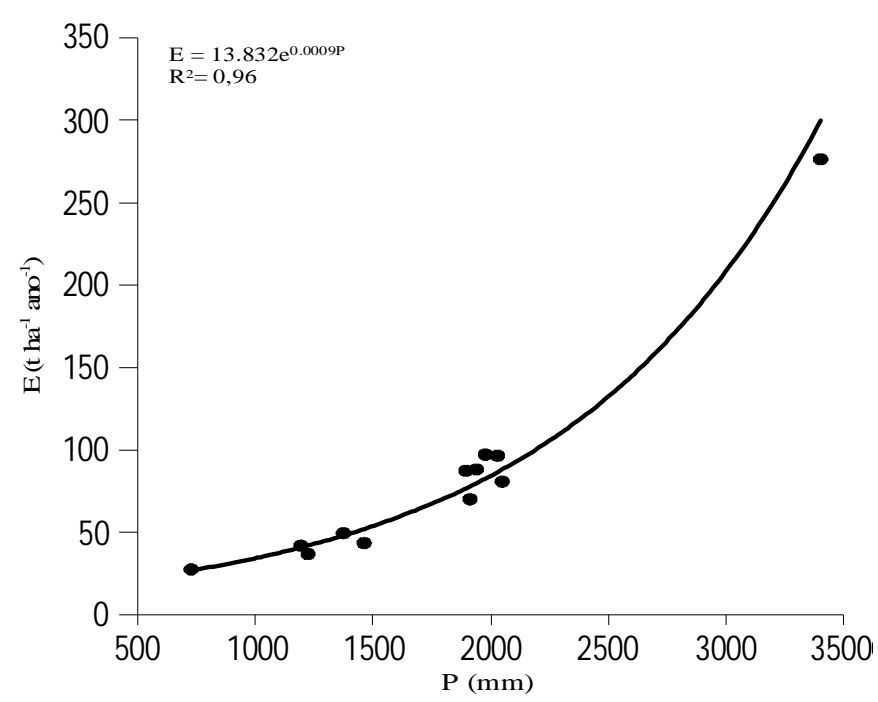

Figura 2. Relação entre a precipitação média anual (P) e a produção de sedimentos anual calculada (E)

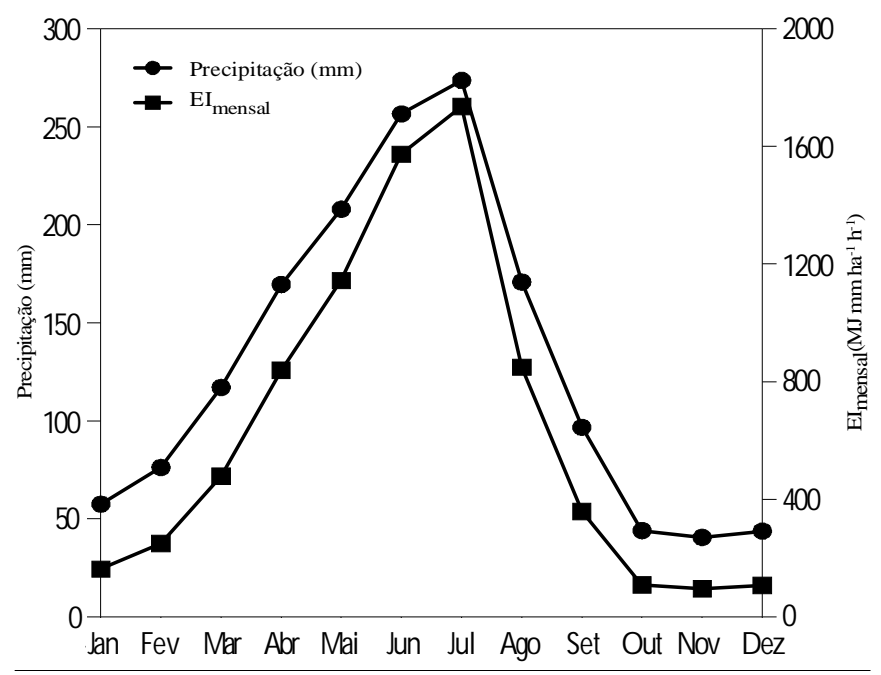

Figura 3. Distribuição dos valores da precipitação média mensal e da erosividade da chuva mensal 

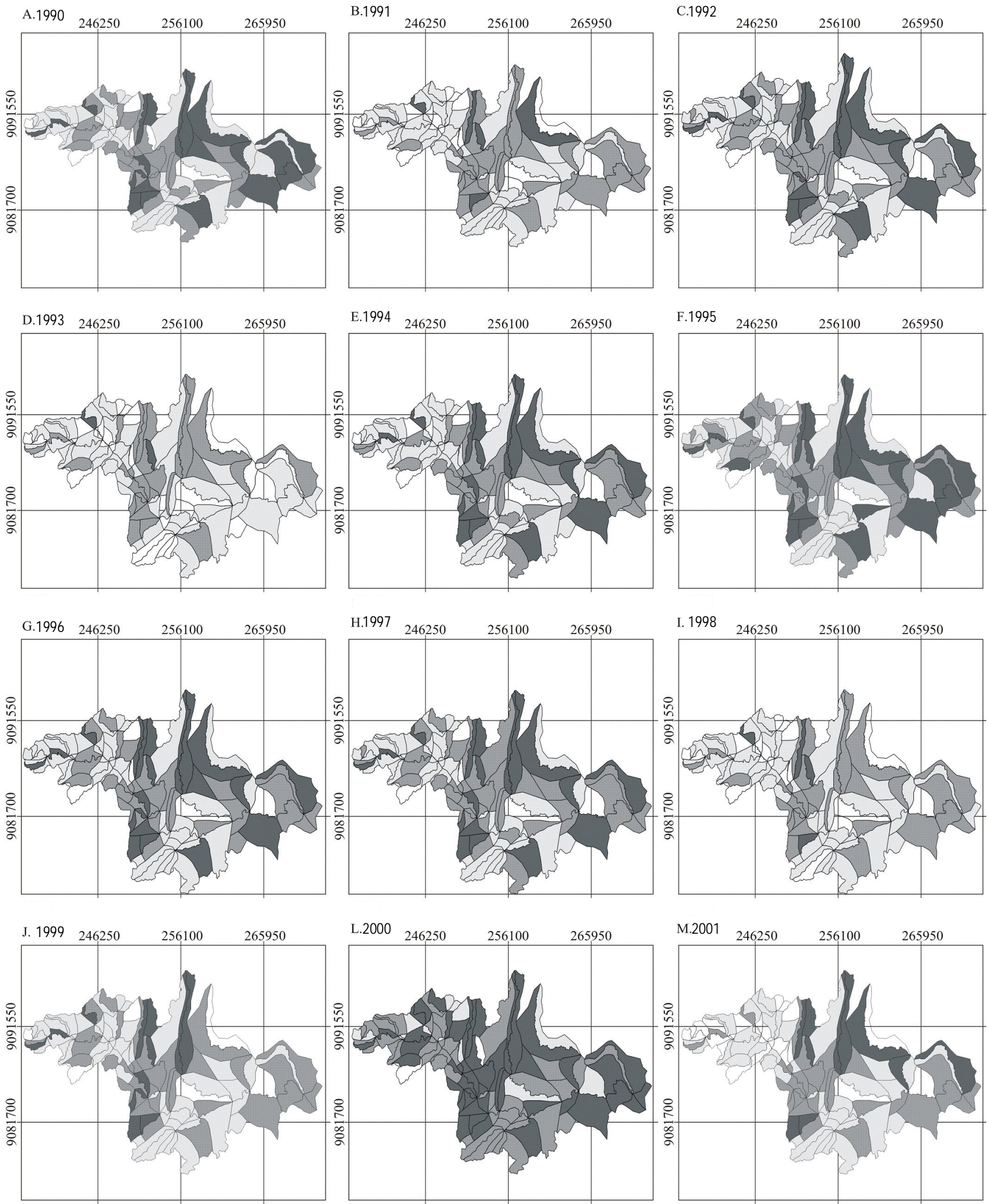

Produtividade média (t ha ${ }^{-1}$ ano $\left.^{-1}\right)$
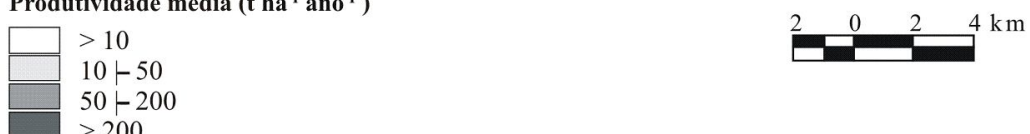

i

Figura 4. Espacialização da produção média calculada de sedimentos para a bacia do rio Pirapama, entre 1990 e 2001 
pelo ajuste mais detalhado, com o maior número possível de parâmetros.

\section{RESULTADOS E DISCUSSÃO}

Os resultados para as simulações da estimativa da produção de sedimentos calculada no exutório da bacia do rio Pirapama para os anos de 1990 a 2001, são apresentados na Tabela 2. Observa-se que a maior produção de sedimentos gerada na bacia está relacionada aos anos com precipitação anual acima da média histórica da região, nos anos de 1995, 1996 e, principalmente, em 2000. Nos anos em que a precipitação média anual foi baixa (1993 e 1998), a produção média anual de sedimentos também foi bem inferior à dos demais anos.

A Figura 1 mostra a produção calculada de sedimentos para a bacia Pirapama numa escala anual, na qual se observa que a produção de sedimentos em 2000 foi superior a $276 \mathrm{t} \mathrm{ha}^{-1}$ ano $^{-1}$ e o volume total de sedimentos produzidos ao longo do período analisado foi aproximadamente de $995 \mathrm{t} \mathrm{ha}^{-1}$.

Analisando-se os resultados da relação entre os valores da precipitação média anual e da produtividade de sedimentos calculada (Figura 2), percebe-se que a produção de sedimentos na bacia se concentrou entre 40 e $100 \mathrm{t} \mathrm{ha}^{-1} \mathrm{ano}^{-1}$ para uma variação de chuva da ordem de 1.100 a $2.100 \mathrm{~mm}^{2} \mathrm{ano}^{-1}$, e o ano de 2000 apresentou disparidade em relação aos demais anos, com valor anual médio de sedimentos da ordem de $276,44 \mathrm{t} \mathrm{ha}^{-1}$ ano $^{-1}$, ou seja, o processo erosivo é muito complexo para ser representado como relação linear simples, dependendo apenas da precipitação média anual, razão pela qual um modelo físico que considera a hidráulica do escoamento e dos sedimentos, deve ser levado em conta.

Por exemplo, quanto ao poder erosivo das chuvas na bacia pode-se constatar, através da comparação dos valores mensais da erosividade da chuva e da precipitação média, apresentados na Figura 3, que os maiores valores médios mensais, tanto da erosividade da chuva quanto da precipitação pluvial na bacia Pirapama, ocorreram nos meses de abril a agosto. A concentração de $79 \%$ da erosividade da chuva em apenas cinco meses é um indicador de que nesse período do ano deve haver medidas preventivas no sentido de proteger o solo da erosão, como mencionado em Albuquerque et al. (2005). Porém, informações mais precisas sobre o evento chuvoso (hietograma) e da bacia (e.g., declividade, rugosidade, tipo de solos) devem ser levadas em consideração pelo modelo, pois as mesmas levariam a resultados diferentes, e por isso os modelos físicos, como o aqui descrito, são incentivados no lugar de modelos conceituais do tipo EUPS.

Para a representação espacial da produção de sedimentos na bacia Pirapama, os resultados da modelagem hidrossedimentológica, calculados para cada plano discretizado, foram inseridos em um SIG e divididos em quatro classes para cada ano analisado: (a) menos de $10 \mathrm{t} \mathrm{ha}^{-1}$ ano $^{-1}$; (b) entre $10 \mathrm{e}$ $50 \mathrm{t} \mathrm{ha}^{-1}$ ano $^{-1}$; (c) entre 50 e $200 \mathrm{t} \mathrm{ha}^{-1} \mathrm{ano}^{-1}$ e (d) mais de $200 \mathrm{t} \mathrm{ha}^{-1}$ ano $^{-1}$. A Figura 4 apresenta a espacialização da produção calculada de sedimentos para cada plano discretizado da bacia, a partir da precipitação diária observada para o período compreendido entre 1990-2001 e implantados no SIG, para a representação espacial da estimativa da produção de sedimentos, segundo as classes de produção de sedimentos mencionadas.

No que tange à identificação das áreas susceptíveis ao processo de erosão, os resultados mostram que as áreas localizadas na parte centro-norte da bacia foram as que mais contribuíram para o processo de erosão se comparadas com os planos inseridos na parte sul da bacia, o que pode ser explicado pelo fato das maiores declividades se localizarem na parte centro-norte da bacia.

De acordo com os resultados apresentados e discutidos na Tabela 2, nos anos com precipitação abaixo da média anual da região (Figura 4D e 4I), a produção de sedimentos foi relativamente mais baixa se comparada com os demais anos analisados; entretanto, as áreas localizadas na parte sul da bacia ainda apresentam índices relativamente altos de erosão.

\section{CONCLUSÕES}

1. Constatou-se que a aplicação do Kineros é viável para ser usado na simulação de eventos contínuos de chuva-vazão e na estimativa da produção de sedimentos em bacias hidrográficas do litoral nordestino com mais de $100 \mathrm{~km}^{2}$; pode ser usado também pelo COBH-Pirapama para estudos futuros de gerenciamento de manejo de solo e água.

2. A integração entre o Kineros e o SIG é uma ferramenta significativa na modelagem da produção de sedimentos e na espacialização dos resultados, para identificação das principais áreas susceptíveis ao processo erosivo da bacia do rio Pirapama.

3. Para a bacia Pirapama estimou-se em cerca de $83 \mathrm{t} \mathrm{ha}^{-1}$ ano $^{-1}$ a perda de solo potencial média na bacia, a qual deve ser considerada alta pois se encontra entre 50 e $200 \mathrm{t} \mathrm{ha}^{-1}$ ano $^{-1}$, segundo a classificação proposta pela FAO (1980).

\section{AGRADECIMENTOS}

Os autores agradecem ao Dr. Carl Unkrich, do Southwesth Watershed Research Center - Estados Unidos, por fornecer o programa fonte utilizado. Os autores foram apoiados com recursos do CT-Hidro/MCT/FINEP, CNPq e CAPES.

\section{LITERATURA CITADA}

Albuquerque, A. W.; Moura Filho, G.; Santos, J. R.; Costa, J. P. V.; Souza, J. L. Determinação de fatores da equação universal de perda de solo nas condições de Sumé, PB. Revista Brasileira de Engenharia Agrícola e Ambiental, v.9, n.2, p.180-188, 2005.

Araújo, J. C.; Knight, D. W. A review of the measurement on sediment yield in different scales. Revista Escola de Minas, v.58, n.3, p.257-265, 2005.

Corradini, C.; Melone, F.; Smith, R. E. Modeling infiltration during complex rainfall sequences. Water Resources Research, v.30, n.10, p.2777-2784, 1994. 
Engelund, F.; Hansen, E. A monograph on sediment transport in alluvial streams. Copenhagen: Teknisk Forlag, 1967, 62p.

FAO - Food and Agriculture Organization. La erosión del suelo por el agua: Algunas medidas para combatirla en las tierras de cultivo. Cuadernos de fomento agropecuário de la Organización de Las Naciones Unidas, Roma: FAO, n.81, 1980, 207p.

Figueiredo, E. E.; Bathurst, J. C. Runoff and sediment yield predictions in a semiarid region of Brazil using SHETRAN. In: IAHS Decade on Prediction in Ungauged Basins, Hydrological Sciences, 2002, Brasília. Resumos ... Brasília: IAHS, 2002. CD Rom.

Firouzabadi, P. Z.; Davoodi, A. Study on soil erosion and sedimentation in Alashtar watershed using image processing software. In: ISPRS Congress, Hydrological Sciences, 2004, Istanbul. Resumos ... Istanbul: ISPRS, 2004. CD Rom.

Kalin, L.; Govindaraju, R. S.; Hantush, M. M. Effect of geomorphologic resolution on modeling of runoff hydrograph and sedimentograph over small watersheds. Journal of Hydrology, v.276, p.89-111, 2003.

Lima, E. R. V.; Kux, H. J. H.; Sausen, T. M. Sistema de informações geográficas e técnicas de sensoriamento remoto na elaboração de mapa de riscos de erosão no sertão da Paraíba. Revista Brasileira de Ciência do Solo, v.16, n. 2, p.257-263, 1992.

Lombardi Neto, F.; Moldenhauer, W. C. Erosividade da chuva: Sua distribuição e relação com perdas de solo em Campinas, SP. Bragantia, v.51, n.2, p.189-196, 1992.
Rawls, W. J.; Brakensiek, D. L.; Saxton, K. E. Estimation of soil water properties. Transactions of the ASAE, v.25, p.1316-1320, 1982.

Santos, C. A. G.; Silva, R. M.; Nitão, I. A. A numerical modeling for surface runoff and sediment yield coupled to a GIS. In: Iberian Latin American Congress on Computational Methods, 25, 2004, Recife. Procedings ... Recife: CILAMCE, 2004. CD Rom.

Santos, C. A. G.; Suzuki, K.; Lopes, W. T. A. Sediment yield in Ishite watershed using a runoff-erosion kinematic model. In: IAHR Congress, 29, 2001, Beijing. Procedings ... Beijing: IAHR, v.2, p.146-151, 2001.

Santos, C. A. G.; Suzuki, K.; Watanabe, M.; Srinivasan, V. S. Influência do tipo da cobertura vegetal sobre a erosão no semi-árido paraibano. Revista Brasileira de Engenharia Agrícola e Ambiental, v.4, n.1, p.92-96, 2000.

Silva, R. M. Previsão hidrossedimentológica numa bacia periurbana através do acoplamento de modelos climáticos e hidrológicos. João Pessoa: UFPB, 2005, 176p. Dissertação Mestrado

Smith, R. E.; Corradini, C.; Melone, F. Modeling infiltration for multistorm runoff events. Water Resources Research, v.29, n.1, p.133-144, 1993.

Woolhiser, D. A.; Smith, R. E.; Goodrich, D. C. Kineros, a kinematic runoff and erosion model: Documentation and user manual. Tucson: U.S. Department of Agriculture, Agricultural Research Service, 1990. 130p. 